\title{
CoNi/Pt interface roughness probed by nonlinear magneto-optics, $x$-ray scattering and atomic force microscopy
}

\author{
K. Bal, ${ }^{\text {a) }}$ A. Kirilyuk, and Th. Rasing \\ Research Institute for Materials, University of Nijmegen, Toernooiveld 1, 6525 ED Nijmegen, \\ The Netherlands \\ Y. Luo and K. Samwer \\ I. Physikalisches Institut, Goettingen University, Bunsenstrasse 9, 37073 Goettingen, Germany \\ M. A. M. Haast and J. C. Lodder \\ MESA Research Institute, University of Twente, 7500 AE Enschede, The Netherlands
}

(Received 15 September 2000; accepted for publication 21 November 2000)

\begin{abstract}
The crystallographic contribution of the nonlinear magneto-optical response from CoNi/Pt interfaces appears to scale linearly with increasing interface roughness as determined by small angle $\mathrm{x}$-ray scattering and atomic force microscopy. From the magnetic contribution it follows that the increased interface roughness causes the interface moment to turn out of plane while the bulk of the film has an in-plane magnetization. (C) 2001 American Institute of Physics.
\end{abstract}

[DOI: 10.1063/1.1342799]

The characteristics of an interface are of critical importance for phenomena like giant magnetoresistance, tunneling magnetoresistance or interlayer exchange coupling found in magnetic ultrathin multilayers with layer thicknesses in the range of $0.5-3 \mathrm{~nm}$. The reason for that is the fact that both electronic and magnetic properties of the interface are crucially dependent on its structure. The establishing of this dependency, however, remains a challenge due to difficulties of existing experimental techniques.

Magnetization-induced second-harmonic generation (MSHG) is a nonlinear magneto-optical technique that has been shown to be a powerful and simple tool by which to study ultrathin magnetic films and multilayers, since it combines a extreme surface/interface sensitivity ${ }^{1,2}$ with very large magneto-optical effects. ${ }^{3}$

Recently, we showed how MSHG could be used to probe the crystallographic and magnetic changes at a $\mathrm{CoNi} / \mathrm{Pt}$ interface induced by changing the sputtering conditions. ${ }^{4}$ The observed changes were assumed to be related to the increasing interfacial roughness with increasing sputtering pressures, as supported by Ref. 5. Here, we prove the direct correlation between the crystallographic contribution of the MSHG technique with the roughness using small angle $\mathrm{x}$-ray scattering (SAXS) and atomic force microscopy (AFM) studies of $\mathrm{CoNi} / \mathrm{Pt}$ multilayers.

In the electric-dipole approximation, SHG is expressed through the second-order polarization $\mathbf{P}(2 \omega)$ induced in a medium by an incident electromagnetic wave $\mathbf{E}(\omega)$ :

$$
p_{i}(2 \omega)=\chi_{i j k}^{(2)} E_{j}(\omega) E_{k}(\omega)
$$

The third-rank polar tensor $\chi^{(2)}$ vanishes in any centrosymmetric medium. Hence, a symmetry breaking surface or interface is a source of SHG, giving rise to the extreme interface sensitivity of the technique. The presence of a mag-

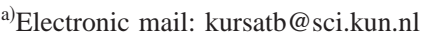

netization does not influence the bulk inversion symmetry but changes the symmetry at the interface, making MSHG probing also interface sensitive. ${ }^{6}$ For an isotropic [or (001)] surface the nonlinear magneto-optical tensor elements contributing to the total second harmonic signal are given in Table I.

The elements shown in boldface are odd in the magnetization (approximately proportional to $\mathrm{it}^{7}$ ) and are called magnetic (magn). The elements that are even in the magnetization, are called crystallographic (cr). For each polarization combination, the even and odd tensor elements can be simplified to two complex effective tensor elements: $\chi_{\mathrm{cr}}^{(2)}$ and $\chi_{\text {magn }}^{(2)}$.

In a multilayer the crucial challenge is how to derive the nonlinear effective tensor elements for each interface. For this, multiple scattering calculations (based on a transfer matrix technique ${ }^{8}$ ) are used to determine the electric field $\mathbf{E}(\omega)$ for every interface; the same calculation procedure is used afterwards to compute the outgoing SHG intensity. The (effective) tensor components $\chi_{i}^{(2)}$ for each interface $i$ are the fitting parameters to describe the experimental data. The interface characteristics, like roughness, degree of interface mixing, or the types of material, are expected to influence the magnitude and polarization of the second harmonic intensity. ${ }^{9,10}$

For the MSHG measurements, a pulsed laser beam from a Ti:sapphire laser $(82 \mathrm{MHz} \times 100 \mathrm{fs}$ pulses $)$ with a wavelength of $840 \mathrm{~nm}$ was focused onto the sample. After proper filtering, the outcoming specular second-harmonic light was detected with a photomultiplier. The asymmetry of the MSHG signal $A=\left(I^{+}-I^{-}\right) /\left(I^{+}+I^{-}\right)$(where $I^{ \pm}$is the MSHG intensity for the magnetization up or down, respectively) was measured as a function of the angle of incidence in the transversal configuration.

The samples, Pt40nm/CoNi3nm/Pt $3 \mathrm{~nm}$ on a $\mathrm{Si}(001)$ substrate, were prepared by sputtering at different Ar pres- 
TABLE I. Nonlinear magneto-optical tensor elements of an isotropic [or (001)] surface in the longitudinal $(\mathbf{M} \| x)$, transversal $(\mathbf{M} \| y)$, and polar (M) magneto-optical configurations ( $x z$ is the plane of incidence).

\begin{tabular}{cccc}
\hline \hline & $P_{\text {in }}$ & $S_{\text {in }}$ & \\
\hline $\mathbf{M} \| x$ & xxz, zxx, zzz & zyy & $P_{\text {out }}$ \\
& $\mathbf{y x x}, \mathbf{y z z}$ & $\mathbf{y y y}$ & $S_{\text {out }}$ \\
$\mathbf{M} \| y$ & xzx, zxx, zzz, & zyy, & $P_{\text {out }}$ \\
& $\mathbf{x x x , ~ x z z , ~ z z x ~}$ & xyy & \\
& $\cdots$ & $\cdots$ & $S_{\text {out }}$ \\
$\mathbf{M} \| z$ & xxz, zxx, zzz & zyy & $P_{\text {out }}$ \\
& yzx & $\cdots$ & $S_{\text {out }}$ \\
\hline \hline
\end{tabular}

sures (between 4 and $36 \mu$ ), with a base pressure of $5 \times 10^{-8}$ mbar. More information on the sample preparation can be found in Ref. 5.

In particular the crystallographic contribution $\chi_{\mathrm{cr}}^{(2)}$ appeared to increase rapidly above $15 \mu$ bar, while staying almost constant below this pressure [see Fig. 1(a)]. This increase was accompanied by a strong decrease of the transversal magnetization component, as obtained from the asymmetry of the MSHG signal. Both were considered to be due to an increasing interfacial roughness for higher pressures. Recently it was shown that at $6 \mu$ bar sputtering $\mathrm{Ar}$ pressure the $\mathrm{CoNi} / \mathrm{Pt}$ multilayers have a low atomic roughness and a strong preferential (111) texture, while at $36 \mu \mathrm{bar}$ the roughness is increased and an additional (200) texture is present. $^{5}$

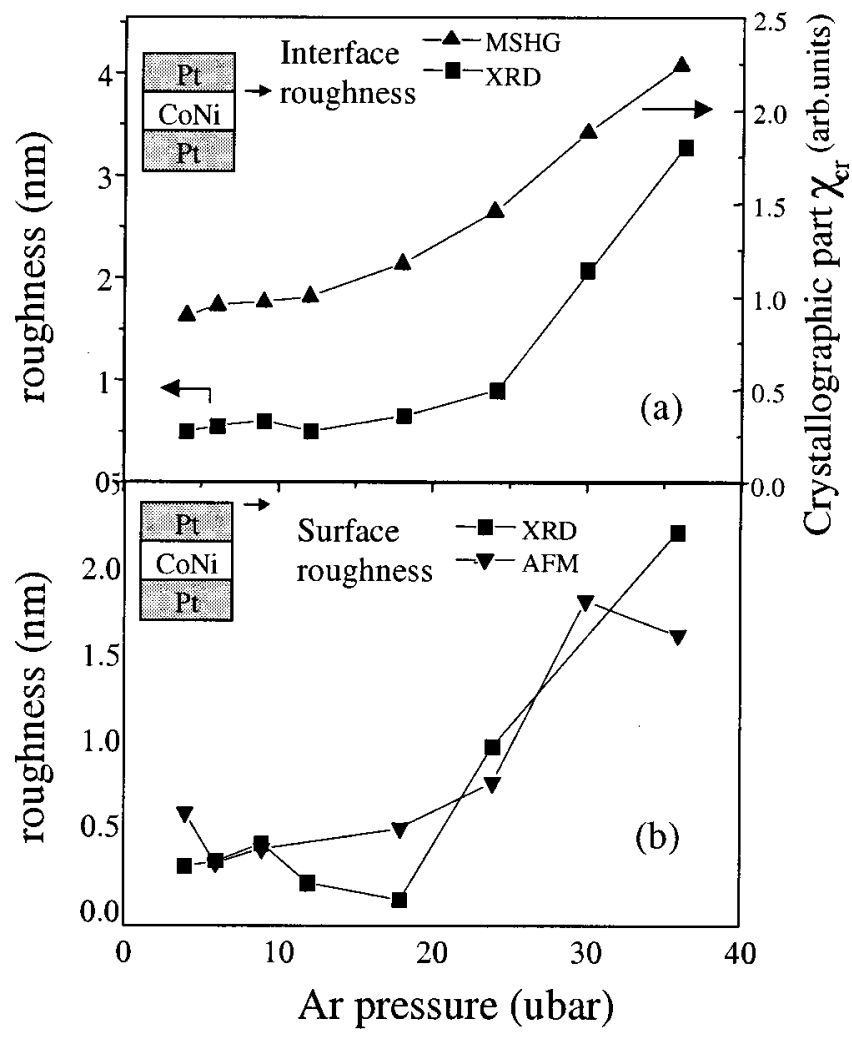

FIG. 1. (a) Crystallographic contribution determined with MSHG and the interface roughness determined with $\mathrm{x}$-ray scattering of the $\mathrm{CoNi} / \mathrm{Pt}$ interfaces as a function of sputtering Ar pressure. (b) Surface roughness of the $\mathrm{CoNi} / \mathrm{Pt}$ sample obtained with small angle $\mathrm{x}$-ray scattering (rectangle) and AFM (circle).

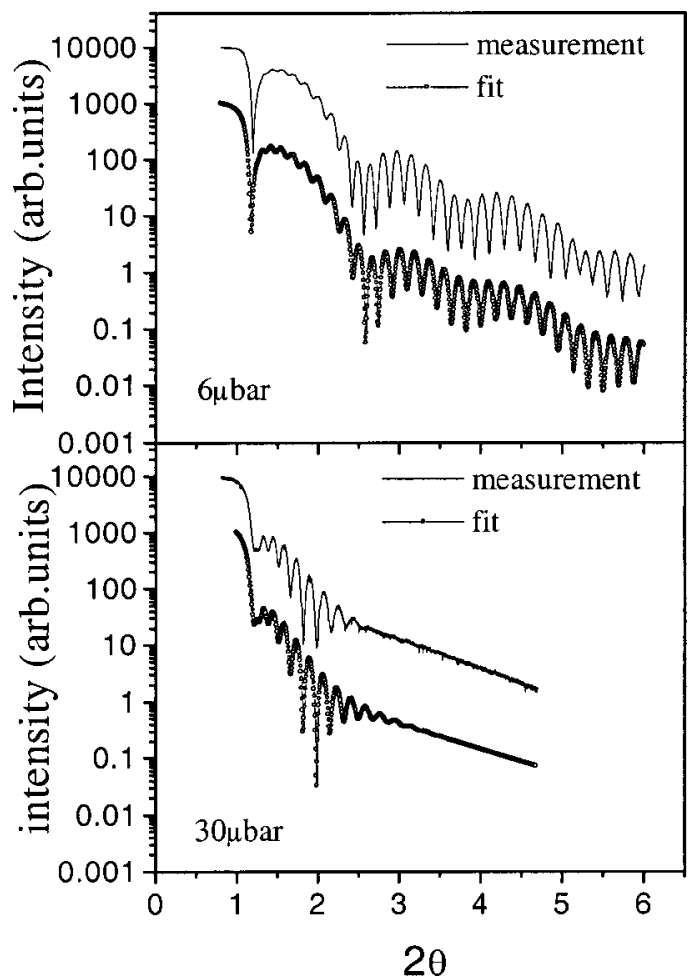

FIG. 2. Measured and fitted small angle x-ray scattering curves for the samples prepared at 6 and $30 \mu$ bar. The measured and fitted curves are shifted for clarity.

To make a clear correlation between the effective $\chi^{(2)}$ elements and the roughness of the buried interfaces we have performed small angle x-ray scattering measurements of all eight samples. The interface roughness of these samples was subsequently obtained by fitting using a multiple reflection model. Measurement and fits of two samples are shown in Fig. 2. Information on similar measurements can be found in Ref. 11. The results are plotted in Fig. 1(a) as a function of sputtering Ar pressure. It can be seen that below $15 \mu$ bar the roughness does not change significantly, while starting from 15 to $20 \mu$ bar the roughness starts to increase, in excellent agreement with the MSHG measurements. Although in Ref. 5 an increase of the (200) texture up to $20 \%$ coverage is observed, this is only expected to be a second-order effect. Because the texture is still dominantly (111) and the grains are randomly oriented (polycrystalline), no significant difference is expected in the polarizability between the two types of surfaces.

As an additional check of the roughness values obtained from the X-ray data, AFM measurements were performed on the same set of samples. In Fig. 1(b) the surface root mean square (rms) roughness as a function of sputtering Ar pressure obtained with AFM and x-ray scattering is shown. The two curves show very good agreement with one another and show a similar dependency as the interface roughness on the sputtering Ar pressure.

From these measurements on the $\mathrm{CoNi} / \mathrm{Pt}$ interface we clearly see that the increase of $\chi_{\mathrm{cr}}^{(2)}$ is correlated with the increasing interfacial roughness (see Fig. 3). For interface roughness below $1 \mathrm{~nm}$, one observes a linear relationship between $\chi_{\mathrm{cr}}^{(2)}$ and the interface roughness, whereas $\chi_{\mathrm{cr}}^{(2)}$ 


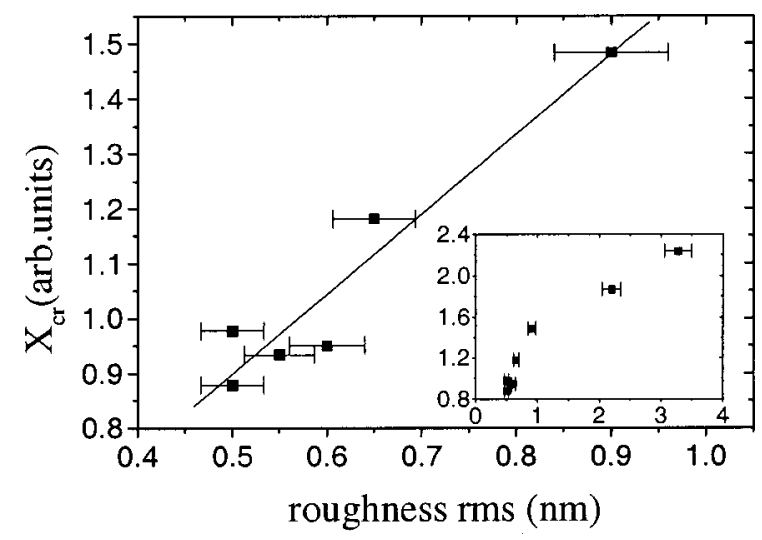

FIG. 3. $\chi_{\mathrm{cr}}^{(2)}$ plotted vs the interface roughness determined from the data in Fig. 1(a).

changes more slowly above $1 \mathrm{~nm}$ (see the inset of Fig. 3). These results are very similar to those obtained for $\mathrm{Si}-\mathrm{SiO}_{2}$ interfaces ${ }^{9,12}$ except for the fact that there the nonlinear susceptibility decreased with increasing roughness. An increase for the SHG response with increasing roughness is more often observed for metallic surfaces and has been interpreted as resulting from excitations of local plasmons. ${ }^{13,14}$ However, more recent work shows that from a microscopic point of view, the presence of many steps and kinks on atomically rough surfaces may enhance the total nonlinear optical response due to the contribution of the step edges. ${ }^{10,15}$

In Ref. 4 it was found that the magnetic contribution has a clear maximum near $15 \mu$ bar (see Fig. 4) after which this contribution decreased with increasing sputtering pressure. This was explained by stating that for low Ar pressures the interface layers become slightly intermixed due to the high energies of sputtered atoms and leads to a lower magnetic contribution. The decrease for larger $p_{\text {Ar }}$ was related to a decrease in the in-plane magnetic moment of the rough interface since the measurement was done in the transversal configuration. The latter suggests that the increasing rough-

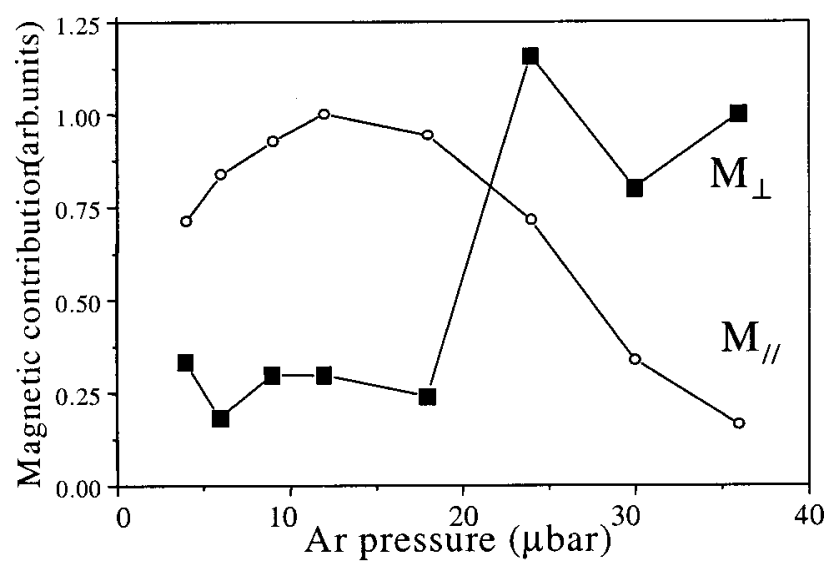

FIG. 4. Magnetic contributions to the MSHG from the CoNi/Pt magnetic interfaces as a function of sputtering Ar pressure with contributions from $\mathbf{M}_{\perp}\left(P_{\text {in }} S_{\text {out }}\right)$ out-of-plane magnetization and $\mathbf{M}_{\|}\left(P_{\text {in }} P_{\text {out }}\right)$ in-plane magnetization. ness may lead to an out-of plane tilt of the local interface magnetic moments by changing the local coordination of the Co atoms. Such a polar magnetization may arise at rough interfaces due to strong perpendicular magnetic interface anisotropy, on the one hand, and due to the possible presence of interface grains that are relatively decoupled from the bulk, on the other.

From Table I, it can be seen that only in the case of the appearance of either a polar and/or longitudinal component the $P_{\text {in }} S_{\text {out }}$ signal is expected to become nonzero. In Fig. 4, the $P_{\text {in }} S_{\text {out }}\left(M_{\perp}\right)$ signal is plotted as a function of Ar pressure, showing a strong increase above $20 \mu$ bar. This is strong support for the appearance of a polar magnetization component. Even if on a small scale there would be local longitudinal magnetization components, on a larger scale laser spot size $(\sim 100 \mu \mathrm{m})$ these would cancel one another due to the in-plane symmetry.

In summary, we have shown that the nonlinear magnetooptical technique of magnetization-induced second-harmonic generation is an excellent tool by which to probe both structural and magnetic properties of buried interfaces. By comparison with SAXS and AFM data we have established that for interface roughnesses below $1 \mathrm{~nm}$, the crystallographic contribution to the MSHG scales linearly with the rms roughness. This interpretation will be tested in further experiments to make an even more general statement. For the case of $\mathrm{Pt} / \mathrm{CoNi} / \mathrm{Pt}$ we found that the in-plane magnetic contribution decreased with increasing interface roughness, accompanied by an increase in the polar component.

The authors thank A. V. Petukhov, H. A. M. van den Berg, and I. L. Lyubchanskii for helpful discussions. Part of this work was supported by the European TMR Network NOMOKE and by the BRITE EURAM Project TUNNELSENSE.

${ }^{1}$ H. A. Wierenga, W. de Jong, M. W. J. Prins, Th. Rasing, R. Vollmer, A. Kirilyuk, H. Schwabe, and J. Kirschner, Phys. Rev. Lett. 74, 1462 (1995).

${ }^{2}$ M. Straub, R. Vollmer, and J. Kirschner, Phys. Rev. Lett. 77, 743 (1996).

${ }^{3}$ B. Koopmans, M. Groot Koerkamp, Th. Rasing, and H. van den Berg, Phys. Rev. Lett. 74, 3692 (1995).

${ }^{4}$ A. Kirilyuk, Th. Rasing, M. A. M. Haast, and J. C. Lodder, Appl. Phys. Lett. 72, 2331 (1998).

${ }^{5}$ M. Haast, thesis, University of Twente, Twente, The Netherlands, 1999.

${ }^{6}$ R.-P. Pan, H. D. Wei, and Y. R. Shen, Phys. Rev. B 39, 1229 (1989).

${ }^{7}$ U. Pustogowa, W. Hübner, and K. H. Bennemann, Phys. Rev. B 48, 8607 (1993).

${ }^{8}$ H. A. Wierenga, M. W. J. Prins, and Th. Rasing, Physica B 204, 281 (1995).

${ }^{9}$ J. I. Dadap, B. Doris, Q. Deng, M. C. Downer, J. K. Lowell, and A. C. Diebold, Appl. Phys. Lett. 64, 2139 (1994).

${ }^{10}$ A. V. Petukhov, Ch. Jakobsen, and K. Pedersen, Surf. Sci. 369, 265 (1996).

${ }^{11}$ Y. Luo, M. Moske, and K. Samwer, Europhys. Lett. 42, 565 (1998).

${ }^{12}$ S. T. Cundiff, W. H. Knox, F. H. Kaumann, K. W. Evans-Lutterodt, M. T. Tang, M. L. Green, and H. M. van Driel, Appl. Phys. Lett. 70, 1414 (1997).

${ }^{13}$ O. A. Aktsipetrov, A. A. Nikulin, V. I. Panov, S. I. Vasil'ev, and A. V. Petukhov, Solid State Commun. 76, 55 (1990).

${ }^{14}$ G. T. Boyd, Th. Rasing, J. R. Leite, and Y. R. Shen, Phys. Rev. B 30, 519 (1984).

${ }^{15}$ Q. J. Jin, H. Regensburger, R. Volmer, and J. Kirschner, Phys. Rev. Lett. 80, 4056 (1998). 